\title{
Vegetation Database of South-Western Kyrgyzstan - the walnut-wildfruit forests and alpine pastures
}

\author{
Peter Borchardt \& Udo Schickhoff
}

\begin{abstract}
The following factsheet provides a brief overview of the Vegetation Database of South Western Kyrgystan (GIVD ID ASKG-001) of the project "The Impact of the Transformation Process on Human-Environmental Interactions in Southern Kyrgyzstan". Within the framework of this project phytosociological relevés of the walnut-fruit forest and adjacent subalpine and alpine pastures has been sampled since 2001. Currently, there are 698 relevés available from the walnut-wildfruit forest region (Arslanbob, Bazaar Korgon Rayon, Jalalabad Oblast, Kyrgyzstan). At present the dataset is used by the members of the study group for analysing quantitative and qualitative effects of varying grazing pressure with respect to site-ecological conditions, vegetation patterns, productivity and grazing impact.
\end{abstract}

Keywords: Central Asian endemic; grazing impact; land use change; phytosociology; subalpine/alpine pasture; vegetation classification; walnut-wildfruit forest.

GIVD Database ID: AS-KG-001

Last update: $2012-05-03$

\section{Vegetation Database of South-Western Kyrgyzstan}

Scope: All available relevés with plot sizes $16-500 \mathrm{~m}^{2}$ of walnut-wildfruit forest and adjacent pastures from the study region (Arslanbob, Bazaar Korgon, Kyrgyzstan).
Status: [NA]
Period: 2001-2009

Database manager(s): Peter Borchardt (borchardt@geowiss.uni-hamburg.de); Udo Schickhoff (schickhoff@geowiss.uni-hamburg.de)

Owner: The Impact of the Transformation Process on Human-Environmental Interactions in Southern Kyrgyzstan, sponsored by the VolkswagenFoundation.

Host: Biogeography \& Landscape Ecology study group, c/o Peter Borchardt, Institute of Geography, University of Hamburg, Bundesstr. 55, 20146 Hamburg, Germany

Web address: http://www.kirgistan.uni-hamburg.de

Availability: according to a specific agreement

Database format(s): Excel

Online upload: no

Online search: no

Publication: Borchardt, P.; Schmidt, M. \& U. Schickhoff (2010) Vegetation Patterns in Kyrgyzstan's Walnut-Fruit Forests under the Impact of Changing Forest Use in Post-Soviet Transformation. Die Erde: 141 (3): 255 - 275.

Plot type(s): normal plots

Non-overlapping plots: 698

Plot-size range: $16-500 \mathrm{~m}^{2}$

Total plot observations: 698

Estimate of existing plots: 698

Completeness: $100 \%$

Countries: KG: $100.0 \%$

Number of sources: 9

Valid taxa: [NA]

Forest: $23 \%$ - Non-forest: aquatic: $0 \%$; semi-aquatic: $0 \%$; arctic-alpine: $77 \%$; natural: $0 \%$; semi-natural: $0 \%$; anthropogenic: $0 \%$

Guilds: all vascular plants: $100 \%$; bryophytes (terricolous or aquatic): $12 \%$

Environmental data: altitude: $100 \%$; slope aspect: 100\%; slope inclination: $100 \%$; microrelief: $35 \%$; soil depth: $100 \%$; surface cover other than plants (open soil, litter, bare rock etc.): $35 \%$; soil pH: $35 \%$; other soil attributes: $35 \%$

Performance measure(s): cover: $100 \%$

Geographic localisation: GPS coordinates (precision $25 \mathrm{~m}$ or less): $88 \%$; point coordinates less precise than GPS, up to $1 \mathrm{~km}$ : $12 \%$; political units or only on a coarser scale $(>10 \mathrm{~km}): 100 \%$

Sampling periods: $2000-2009: 100.0 \%$

Information as of 2012-07-12; further details and future updates available from http://www.givd.info/ID/AS-KG-001

Peter Borchardt* (borchardt@ geowiss.uni-hamburg.de), Udo Schickhoff (schickhoff@ geowiss.uni-hamburg.de) Institute of Geography, University of Hamburg, Bundesstr. 55, 20146 Hamburg, GERMANY

*Corresponding author 